\title{
Resolving Occlusion Method of Virtual Object in Simulation Using Snake and Picking Algorithm
}

\author{
JeongHee Cha, GyeYoung Kim, and HyungIl Choi \\ Information and media institute, School of Computing, School of Media, \\ Soongsil University, Sangdo 5 Dong, DongJak Gu, Seoul, Korea \\ pelly@vision.ssu.ac.kr, \{gykim1,hic\}@ssu.ac.kr
}

\begin{abstract}
For realistic simulation, it is essential to register the two worlds, calculate the occlusion realm between the real world and the virtual object, and determine the location of the virtual object based on the calculation. However, if the constructed map is not accurate or the density is not sufficient to estimate the occlusion boundary, it is very difficult to determine the occlusion realm. In order to solve this problem, this paper proposes a new method for calculating the occlusion realm using the snake and picking algorithm. First, the wireframe generated by the CCD image and DEM was mapped using the visual clues to acquire 3D information in the experimental realm, and the 3D information was calculated at the point where occlusion problem for a moving target. The validity of the proposed approach under the environment in which partial occlusion occurs has been provided by an experiment.
\end{abstract}

Keywords: Occlusion, Snake, Picking, DEM, Augmented Reality, Simulation.

\section{Introduction}

Augmented reality is an area of technology that has originated in virtual reality. While virtual reality offers a virtual world in which users are completely immersed, augmented reality offers virtual objects on the basis of real world images. At present, augmented reality technology is being researched and applied to various areas including the military, medicine, education, construction, game, and broadcasting. This paper studied on the development of a realistic simulated training model through the display of virtual targets in the input images of CCD camera mounted in a tank and the determination of occlusion areas generated from the creation and movement of virtual objects through a movement path according to a scenario. Augmented reality has three general characteristics: image registration, interaction, and real time[1]. Image registration refers to the matching of the locations of the real world object that users watch and the related virtual object, real time refers to the real time image registration and interaction. Interaction implies that the combination of virtual objects and the objects in real images must be harmonized with surrounding environment in a realistic manner, and refers to the determination of occlusion areas according to the changed location or line of sight of the observer or the re-rendering of virtual objects after detection of collisions. However, to solve the problems of 
occlusion such as the hiding of farther virtual objects by closer objects and the covering of objects in real images by other objects, the two worlds must be accurately coordinated and then the depth of the actual scene must be compared with the depth of virtual objects[2][3]. But if the accuracy or density of the created map is insufficient to estimate the boundary of occlusion area, it is difficult to determine the occlusion area. To solve this problem, first, we created a 3D wireframe using the DEM of the experiment area and then coordinate this using CCD camera images and visual clues. Second, to solve the problem of occlusion by accurately estimating the boundary regardless of the density of map, this paper also proposed a method to obtain the reference 3D information of the occlusion points using the Snake algorithm and the Picking algorithm and then to infer the 3D information of other boundaries using the proportional relations between 2D and 3D DEMs. Third, for improving processing speed, we suggest a method by comparing the MER(Minimum Enclosing Rectangle) area of the object in the camera's angle of vision and the MER of the virtual target. Fig. 1 shows the proposed system framework.

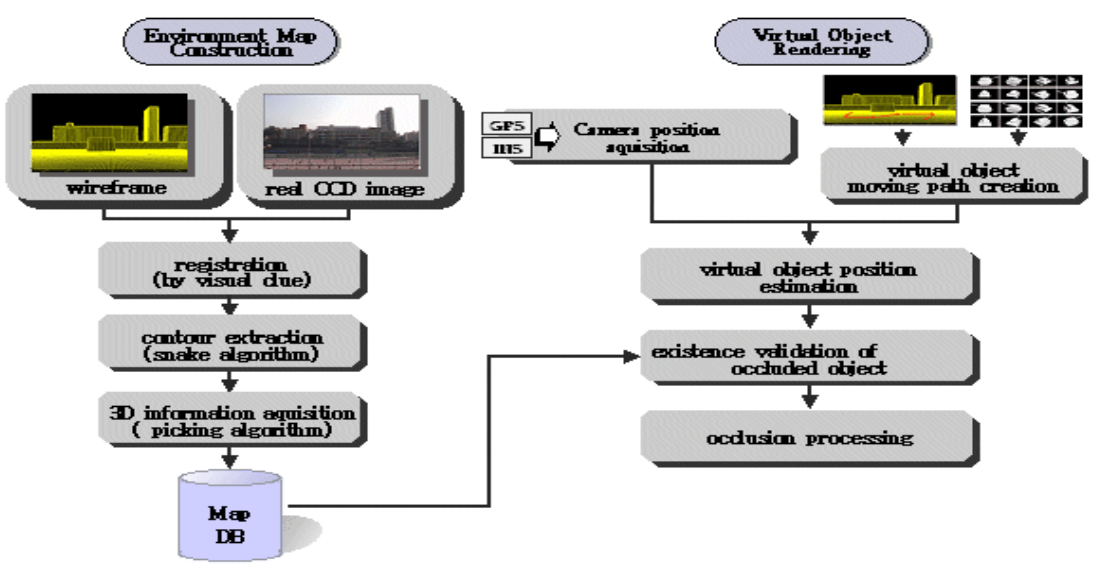

Fig. 1. Proposed System Framework

\section{Methodology}

\subsection{Formation of Wireframe Using DEM and Registration with Real Images Using Visual Clues}

The topographical information DEM (Digital Elevation Model) is used to map the real world coordinates to each point of the 2D CCD image. DEM has information on the latitude and longitude coordinates expressed in $\mathrm{X}$ and $\mathrm{Y}$ and heights in fixed interval. The DEM used for this experiment is a grid-type DEM which had been produced to have the height information for $2 \mathrm{D}$ coordinates in $1 \mathrm{M}$ interval for the limited experiment area of $300 \mathrm{~m}$ x $300 \mathrm{~m}$. The DEM data are read to create a mesh 
with the vertexes of each rectangle and a wireframe with $3 \mathrm{D}$ depth information as Fig. 2[4][5]. This is overlaid on the sensor image to check the coordination, and visual clues are used to move the image to up, down, left or right as shown in Fig. 3, thus reducing error. Based on this initial coordinated location, the location changes by movement of vehicles were frequently updated using GPS (Global Positioning System) and INS (Inertial Navigation System).
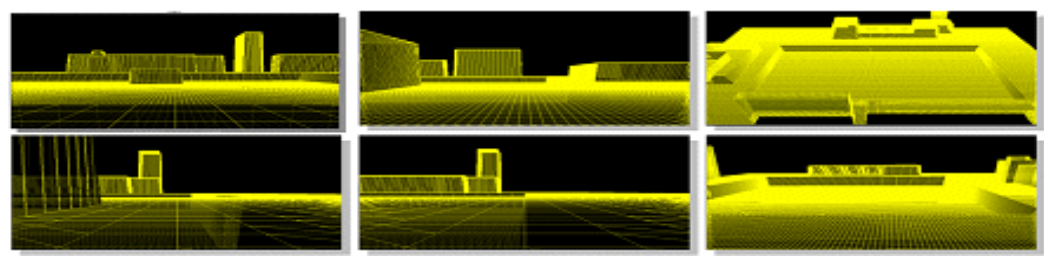

Fig. 2. Wireframe Creation using DEM

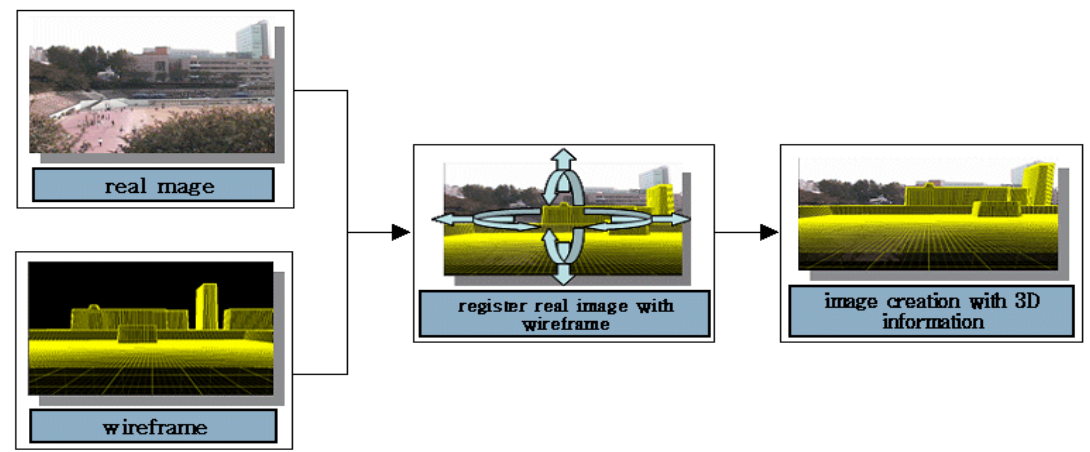

Fig. 3. Registration of Two Worlds using Visual Clues

\subsection{Extracting the Outline of Objects and Acquiring 3D Information}

The Snake algorithm[6][7] is a method of finding the outline of an object by repeatedly moving to the direction of minimizing energy function from the snake vertex input by user. The energy function is shown in Expression [1]. As the energy function is calculated for a discrete space, the parameters of each energy function become the coordinates of each vertex in the image. In Expression [1], $v(s)$ is the snake point, and $v(s)=(x(s), y(s))$, where $x(s)$ and $y(s)$ refer to the positions of $x$ and $y$ in the image of the snake point. Also, $\alpha, \beta$ and $\gamma$ are weights, and this paper gave $\alpha=1, \beta=0.4$, and $\gamma=2.0$, respectively.

$$
E_{\text {snake }}=\int_{0}^{1}\left(\alpha E_{\text {cont }}(v(s))+\beta E_{\text {curve }}(v(s))+\gamma E_{\text {image }}(v(s))\right) d s
$$


The first term is the energy function that represents the continuity of the snake vertexes surrounding the occlusion area and the second term is the energy function that controls the smoothness of the curve forming the snake, of which the value increases along with the curvature, enabling the detection of corner points. Lastly, $E_{\text {image }}$ is a image feature function. All energy functions are normalized to have a value between 1 and 0 . As shown in Table 1, this algorithm extracts the outline by repeatedly performing the energy minimization algorithm which sets a 3 pixels $x 3$ pixels window at each vertex $v(i)$, finds positions where energy is minimized in consideration of the continuity between previous and next vertexes, curvature, and edge strength, and then moves the vertexes to the positions.

Table 1. Snake Algorithm

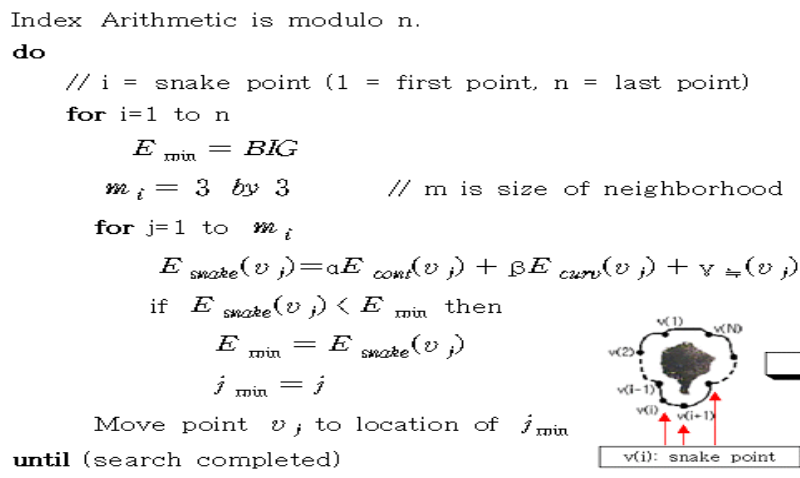

\subsection{Acquisition of 3D Information Using the Picking Algorithm}

In order to acquire the 3D information of the extracted vertexes, this paper used the Picking algorithm which is a well-known 3D graphics technique[8]. It finds the collision point with the 3D wireframe created by DEM that corresponds to the points in $2 \mathrm{D}$ image and provides the $3 \mathrm{D}$ information of the points. The picking search point is the lowest point of the vertexes of the objects extracted from the $2 \mathrm{D}$ image. The screen coordinate system that is a rectangular area indicating a figure that has been projection transformed in the $3 \mathrm{D}$ image rendering process must be converted to the viewport coordinate system in which the actual 3D topography exists to pick the coordinate system where the mouse is actually present. First, the conversion matrix to convert viewport to screen is used to obtain the conversion formula from $2 \mathrm{D}$ screen to $3 \mathrm{D}$ projection window, and then the ray of light is lengthened gradually from the projection window to the ground surface to obtain the collision point between the point to search and the ground surface. Fig. 4 is an example of picking the collision point between the ray of light and DEM. The lowest point of the occlusion area indicated by an arrow is the reference point to search, and this becomes the actual position value of $2 \mathrm{D}$ image in a $3 \mathrm{D}$ space. 

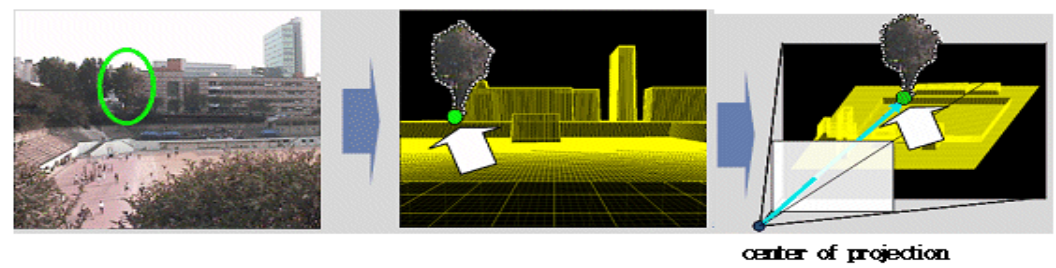

(a)occlusion candidate (b)matching ref.point and DEM (c)3D information extraction

Fig. 4. 3D information Extraction using Collision point of Ray and DEM

\subsection{Creation of 3D Information Using Proportional Relational Expression}

The collision point, or reference point, has 3D coordinates in DEM, but other vertexes of the snake indicated as object outline cannot obtain 3D coordinates because they don't have a collision point. Therefore, this paper suggested obtaining a proportional relation between 2D image and 3D DEM using the collision reference point and then obtaining the 3D coordinates of another vertex. Fig. 5 shows the proportional relation between 2D and 3D vertexes. In Fig. 5, $S_{m}$ is center of screen, $S_{B}$ is reference point of snake vertex (lowest point), $\Delta S_{B}=\left(\Delta S_{x_{B}}, \Delta S_{y_{B}}\right), S_{k}$ is a point except reference point, $\Delta S_{k}=\left(\Delta S_{x_{k}}, \Delta S_{y_{k}}\right) . P_{m}$ is projection point of straight line of $P_{B}$ in 3D, which is through the center of screen. $P_{B}$ is 3D correspondence point of $S_{B}$, $\Delta P_{B}=\left(\Delta P_{x_{B}}, \Delta P_{y_{B}}, \Delta P_{z_{B}}\right), \quad P_{k} \quad$ is a point except reference point, $\Delta P_{k}=\left(\Delta P_{x_{k}}, \Delta P_{y_{k}}, \Delta P_{z_{k}}\right), t=P_{o} P_{B}, t_{m}=P_{o} P_{m}, \theta_{B}: \angle t t^{\prime}, \phi_{B}: \angle t^{\prime} t_{m} . t^{\prime}$ is projected vector of $t$ to $x z$ plane.
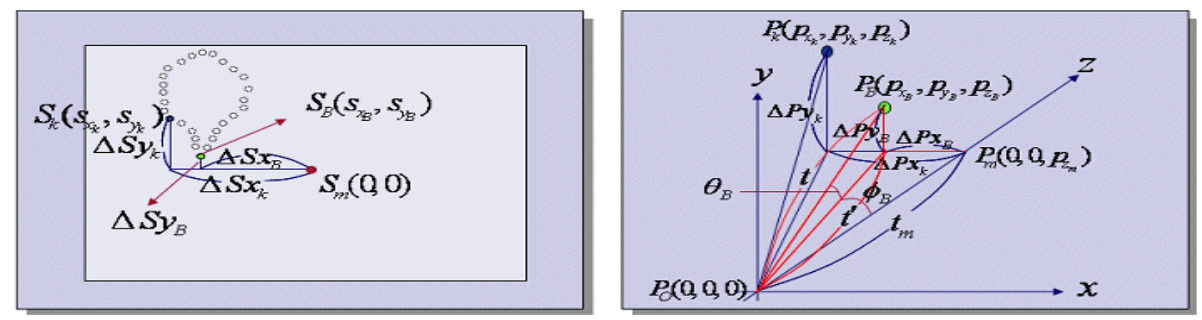

Fig. 5. Proportional Relation of the Vertex in 2D and 3D

To get $p_{m}$ that passes the center of the screen using the coordinates of the reference point obtained above, ${ }^{\prime}$ must be obtained first. As the ${ }^{t}$ value is given by 
the picking ray, the given $t$ value and $y_{B}$ are used to get $\theta_{B}$ and $t^{\prime}$ is obtained using this $\theta_{B}$ in Expression (2).

$$
\theta_{B}=\sin ^{-1}\left(\frac{\Delta P_{y_{B}}}{t}\right), \quad t^{\prime}=\left|t_{B}\right| \cos \left(\theta_{B}\right) \quad t^{\prime}=\left|t^{\prime}\right|
$$

To get $t_{m}, \phi_{B}$ is obtained from Expression (2) which is the angle between t' and $\mathrm{t}_{\mathrm{m},} t_{m}$ can be obtained using $\phi_{B}$ from Expression (3).

$$
\phi_{B}=\tan ^{-1}\left(\frac{\Delta P_{x_{B}}}{t^{\prime}}\right), t^{\prime}=\left|t_{m}\right| \cos \left(\phi_{B}\right),\left|t_{m}\right|=\frac{\left|t^{\prime}\right|}{\cos \left(\phi_{B}\right)} \quad t_{m}=\left|t_{m}\right|
$$

Because $t_{m}=p_{z_{m}}, P_{m}=\left(0,0, t_{m}\right)$.

Now, we can present the relation between the 2D screen view in Fig. 5 and the 3D space coordinates, and this can be used to get $p_{k}$, which corresponds to the $2 \mathrm{D}$ snake vertex.

$$
\begin{gathered}
\Delta S_{B}: \Delta P_{B}=\Delta S_{k}: \Delta P_{k}, \Delta S_{x_{B}}: \Delta P_{x_{B}}=\Delta S_{x k}: \Delta P_{x_{k}}, \\
\Delta P_{x_{k}}=\frac{\Delta P_{x_{B}} \times \Delta S_{x_{k}}}{\Delta S_{x_{B}}}, \Delta S_{y_{B}}: \Delta P_{y_{B}} \Delta S_{y k}: \Delta P_{y_{k}}, \Delta P_{y_{k}}=\frac{\Delta P_{y_{B}} \times \Delta S_{y_{k}}}{\Delta S_{y_{B}}}
\end{gathered}
$$

Consequently, we can get $\Delta P_{k}=\left(\Delta P_{x_{k}}, \Delta P_{y_{k}}\right)$, which is the 3D space point corresponding to each snake vertex to search.

\subsection{Creation of Virtual Target Path and Selection of Candidate Occlusion Objects Using MER (Minimum Enclosing Rectangle)}

To test the proposed occlusion-resolving algorithm, we created the movement path of a virtual target, and determined the changes of the direction and shape of the target as well as the 3D position of the target. First, the beginning and end points of the target set by instructor were saved and the angle of these two points was calculated, and the direction and shape of the target were updated in accordance with the change of the angle. Further, the remaining distance was calculated using the speed and time of the target, and the 3D coordinates corresponding to the position after movements were determined. We also suggest a method of improving processing speed by comparing the MER (Minimum Enclosing Rectangle) area of the object in the camera's angle of vision and the MER of the virtual target because the relational operations between all objects extracted from the image for occlusion processing and the virtual target take much time. The MER (Minimum Enclosing Rectangle) of an object refers to the 
minimum rectangle that can enclose the object and determines the object that has an overlapping area by comparing the objects in the camera image and the MER of the virtual target. In addition, the distance between object and virtual target is obtained using the fact that the determined object and virtual target are placed more or less in a straight line from the camera, and this value was used to determine whether there exists an object between the virtual target and the camera.

\section{Experimental Results}

Fig. 6(left) shows movement path of the virtual target which trainee sets. Also, (right) shows the various virtual targets created to display the targets changing with movement on the image.
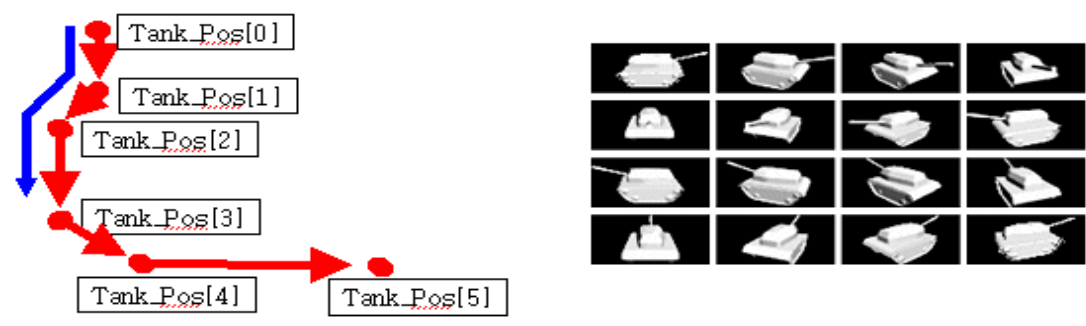

Fig. 6. Moving Route Creation(left) and Appearance of Virtual Object as it Moved(right)

Fig. 7 shows the virtual images moving along the path by frame. We can see that as the frames increase, it is occluded between the tank and the object.

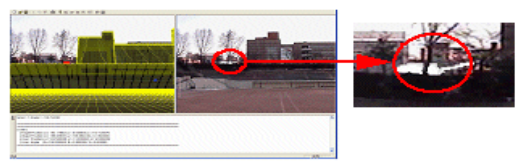

(a) 61 Frame

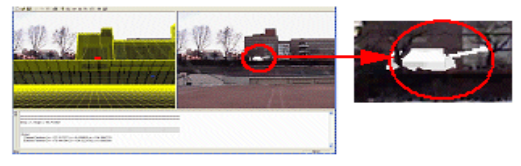

(c) 155 Frame
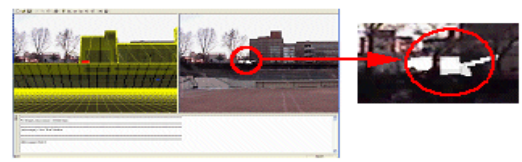

(b) 102 Frame

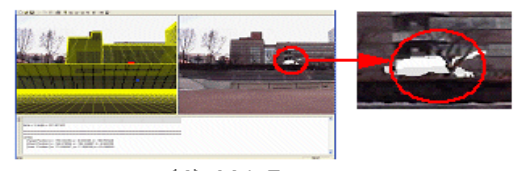

(d) 301 Frame

Fig. 7. Experimental Results of Moving and Occlusion

Table 2 compares between the case of using snake vertexes to select objects in the image to compare with virtual targets and the case of using the proposed MER. With the proposed method, the processing speed decreased by 1.671, which contributed to performance improvement. 
Table 2. Speed Comparison

\begin{tabular}{lcccc}
\hline Method & Total frame & Used object & Speed $(\mathrm{sec})$ & Frame per sec. \\
\hline Snake vertexes & 301 & 10 & 112 & 2.687 \\
MER(proposed) & 301 & 10 & $67 \ldots$ & 4.492 \\
\hline
\end{tabular}

\section{Conclusions}

To efficiently solve the problem of occlusion that occurs when virtual targets are moved along the specified path over an actual image, we created 3D virtual world using DEM and coordinated this using camera images and visual clues. Moreover, the Snake algorithm and the Picking algorithm were used to extract an object that is close to the original shape to determine the $3 \mathrm{D}$ information of the point to be occluded. To increase the occlusion processing speed, this paper also used the method of using the 3D information of the MER area of the object, and proved the validity of the proposed method through experiment. In the future, more research is required on a more accurate extracting method for occlusion area that is robust against illumination as well as on the improvement of operation speed.

\section{Acknowledgement}

This work was supported by the Korea Research Foundation Grant funded by the Korean Government(MOEHRD)(KRF-2006-005-J03801).

\section{References}

[1] Bimber, O. and Raskar, R.,Spatial Augmented Reality: A Modern Approach to Augmented Reality, Siggraph 2005, Los Angeles USA

[2] J. Yong Noh and U. Neumann. Expression cloning. In SIGGRAPH'01, pages 277-288, 2001.

[3] E. Chen. Quicktime VR-an image-based approach to virtual environment navigation. Proc. of SIGGRAPH, 1995.

[4] Lilian Ji, Hong Yan, "Attractable snakes based on the greedy algorithm for contour extraction", Pattern Recognition 35, pp.791-806 (2002)

[5] Charles C. H. Lean, Alex K. B. See, S. Anandan Shanmugam, "An Enhanced Method for the Snake Algorithm," icicic, pp. 240-243, First International Conference on Innovative Computing, Information and Control - Volume I (ICICIC'06), 2006

[6] Wu, S.-T., Abrantes, M., Tost, D., and Batagelo, H. C. 2003. Picking and snapping for 3d input devices. In Proceedings of SIBGRAPI 2003, 140-147. 